\title{
The Effect of Lunar-like Satellites on the Orbital Infrared Light Curves of Earth-analog Planets
}

\author{
Nicholas A. Moskovitzl \\ Institute for Astronomy \\ University of Hawaii at Manoa \\ 2680 Woodlawn Drive \\ Honolulu, HI 96822, USA \\ Email:nmosko@ifa.hawaii.edu \\ Phone: (808) 956-6700 \\ Fax: (808) 956-2901
}

Eric Gaidos

Department of Geology and Geophysics

University of Hawaii at Manoa

1680 East-West Road

Honolulu, HI 96822, USA

Darren M. Williams

School of Science

Penn State Erie, The Behrend College

Erie, PA 16563, USA

Running title: IR Light Curves of Planets with Large Satellites

$\overline{{ }^{1} \text { Corresponding Author }}$ 


\begin{abstract}
We investigate the influence of lunar-like satellites on the infrared orbital light curves of Earth-analog extra-solar planets. Such light curves will be obtained by NASA's Terrestrial Planet Finder (TPF) and ESA's Darwin missions as a consequence of repeat observations to confirm the companion status of a putative planet and to determine its orbit. We use an energy balance model to calculate disk-averaged infrared (bolometric) fluxes from planet-satellite systems over a full orbital period (one year). The satellites are assumed to lack an atmosphere, have a low thermal inertia like that of the Moon and span a range of plausible radii. The planets are assumed to have thermal and orbital properties that mimic those of the Earth while their obliquities and orbital longitudes of inferior conjunction remain free parameters. Even if the gross thermal properties of the planet can be independently constrained (e.g. via spectroscopy or visible-wavelength detection of specular glint from a surface ocean) only the largest ( Mars-size) lunar-like satellites can be detected by light curve data from a TPF-like instrument (i.e. one that achieves a photometric signal-to-noise of 10-20 at infrared wavelengths).

Non-detection of a lunar-like satellite can obfuscate the interpretation of a given system's infrared light curve so that it may resemble a single planet with high obliquity, different orbital longitude of vernal equinox relative to inferior conjunction and in some cases drastically different thermal characteristics. If the thermal properties of the planet are not independently established then the presence of a lunar-like satellite cannot be inferred from infrared data, thus demonstrating that photometric light curves alone can only be used for preliminary study and that the addition of spectroscopic data will be necessary
\end{abstract}


to properly characterize extra-solar Earth-like planets.

Keywords: Planetary systems - planets and satellites: general - astrobiology - methods: data analysis 


\section{INTRODUCTION}

Planets with a minimum mass of 5-6 Earth masses have recently been detected around low mass stars (Udry et al., 2007; Rivera et al., 2005) and it seems likely that observatories such as CoRoT or Kepler will detect yet smaller planets (Gillon et al., 2005). Space-based observatories of the future will be capable of directly detecting Earth-sized planets around other stars. Proposed missions include a coronagraph operating at visible wavelengths (TPFC) (Traub et al., 2006), and a large-baseline interferometer operating in the infrared (TPF-I and Darwin) (Beichman et al., 2006; Fridlund, 2000). One goal of such missions is to distinguish between planets that are Earth-like and can support life, and those that are decidedly less so (e.g., analogs to Mercury, Venus, or Mars). Several techniques have been proposed to carry out this classification. Spectroscopy can reveal the presence of atmospheric gases such as $\mathrm{H}_{2} \mathrm{O}, \mathrm{CH}_{4}$ and $\mathrm{O}_{2}$, which are indicative of temperate conditions and/or biological activity (Des Marais et al., 2002). Photometry in reflected light can reveal diurnal (rotational) variability associated with ice, oceans, land and vegetation across the surface of a planet if no clouds are present (Ford et al., 2001). The specular "glint" from oceans might be detected as an increase in the visible flux and polarization of reflected light at large phase angles (Williams \& Gaidos, 2008; McCullough, 2008). Selsis (2004) showed that orbital infrared light curves could reveal general thermal properties of terrestrial planets. Gaidos \& Williams (2004, hereafter GW04) showed that diurnally averaged, orbital light curves at thermal infrared wavelengths contain information about the thermal properties of the planet's emitting layer (surface or clouds) and obliquity. Such light curves would be generated as a 
byproduct of repeated observations to confirm the companion status and orbit of a putative planet and function as a first step towards characterization. One finding of GW04 was that oceans or a thick atmosphere damp seasonal variations in temperature, and that low or no variability in a planet's infrared light curve is indicative of the presence of oceans or a thick atmosphere. In conjunction with other characteristics, this is a signature of habitable surface conditions. These authors and the work presented here do not consider the effects of variable cloud cover.

The disk-averaged infrared flux of an orbiting planet can vary as it presents different phases to a distant observer. This phenomenon has been observed for Jupiter-mass extra-solar planets with semi-major axes much less than 1 AU (Harrington et al., 2006; Cowan et al., 2007; Knutson et al., 2007). The variation in flux from a planet depends on the diurnal pattern of outgoing infrared flux from the emitting surface (either the top of the atmosphere, if any is present, cloud layers, or the surface), which is controlled by the planet's thermal properties and day length. In general, significant day-night temperature differences will occur only if

$$
c \omega \lesssim\left(\frac{\partial I}{\partial T}\right)_{\bar{T}}
$$

where $c$ is the heat capacity of the surface/atmosphere, $\omega$ is the angular rotation rate, and the right hand side is the slope of the outgoing bolometric infrared flux at the emitting surface vs. temperature, $T$, evaluated at the mean surface temperature of the body, $\bar{T}$. For the Earth: $c=8.34 \times 10^{7} \mathrm{~J} \mathrm{~m}^{-2} \mathrm{~K}^{-1}$ and $\partial I / \partial T=1.58 \mathrm{~W} \mathrm{~m}^{-2} \mathrm{~K}^{-1}$ for $\bar{T}=288 \mathrm{~K}$ (however the effective emitting temperature of the Earth is $255 \mathrm{~K}$ ). Thus equation 1 does not hold for the 
Earth: the Earth's day-night temperature variation is small and would be for any Earth-like planet with a rotation period much less than a year. This is primarily due to the high heat capacity of the ocean mixed layer which also moderates surface temperatures over landmasses and controls the outgoing infrared flux budget.

The primordial rotation periods of terrestrial planets are thought to be a stochastic outcome of the final stages of formation by accretion of planetary embryos, and will be on the order of hours to days (Lissauer et al., 2000). As a consequence, the disk-averaged infrared flux from an Earth-like planet will only vary significantly along the orbit if the planet has a non-zero obliquity or eccentricity and hence seasons. This was explored in GW04.

The Earth's Moon, lacking an atmosphere or oceans and having a lunar day 29.5 times longer than the Earth, experiences a much larger diurnal surface temperature variation. Absence of recent geologic activity on the Moon has allowed a regolith of impact ejecta to accumulate. This material is optically dark [the average lunar Bond albedo is 0.07 (Lane \& Irvine, 1973) compared to the Earth's 0.31] and has a relatively low heat capacity [that of the Moon is $4 \times 10^{4} \mathrm{~J} \mathrm{~m}^{-2} \mathrm{~K}^{-1}$ at 29.5 days or $0.1 \%$ of the Earth, (Muller \& Lagerros, 1998)]. As a result, the inequality of Equation 1 is satisfied and thus the Moon makes a significant or even dominant contribution (depending upon viewing geometry) to the variable component of the infrared flux from the Earth-Moon system.

The Moon is thought to have accreted from a circumterrestrial disk of ejecta generated by the impact of a Mars-sized body (Hartmann, 1986). The high ambient temperatures and low gravity in the transient disk explain the 
Moon's lack of volatiles (Pritchard \& Stevenson, 2000). Current scenarios for the final stages of terrestrial planet formation include such giant impacts (Canup \& Agnor, 1998) and the results of numerical simulations suggest that they are not rare (Ida, Canup \& Stewart, 1997). Thus large satellites lacking atmospheres or oceans may be common around extra-solar rocky planets. Like the Moon, these satellites would have originally formed closer to their parent planets and their rotations would have quickly synchronized to their orbits (Gladman et al., 1996; Canup \& Agnor, 1998). As a result their diurnal temperature variation could be significant. We note that Moon-size satellites could retain an atmosphere against gravitational escape over Gyr time-scales if one was originally present.

Satellites around extra-solar planets will be unresolved by even the most ambitious planet-finding mission: the angular separation of the Earth and Moon at a distance of $10 \mathrm{pc}$ is 0.25 mas. However, a large satellite might reveal itself by a significant variation in the total (bolometric) flux from the system. Such an interpretation requires independent knowledge of the thermal and rotational properties of the parent planet, which can be established using spectroscopy (Des Marais et al., 2002; Selsis, 2004) or optical light curve data (Williams \& Gaidos, 2008; McCullough, 2008). If establishment of these gross thermal properties leads to the expectation that infrared flux variation would be small (Equation 1) then observation of significant variation could be attributed to the presence of a large satellite. In the absence of such auxiliary information, however, the satellite contribution may result in an assignment of erroneous thermal properties to the planet. 
We present calculations of infrared light curves of an Earth-like planet with a Moon-like satellite. The terms Earth-like or Earth-analog refer to specific thermal and orbital properties that represent those of the Earth (see \$21). The albedo, heat capacity and orbital period of the satellite are set to that of the Moon (see above) while its radius is allowed to vary.

In $₫ 2$ we describe the details of the analytical energy balance model (EBM) used in these calculations. We give an illustrative calculation in $\oint_{3}$. We then determine the minimum radius of lunar-like satellite that can be detected at infrared wavelengths around an Earth-analog planet (\$4). In $\$ 5$ we describe the biases in planetary orbital properties that can be introduced by an undetected lunar-like satellite. In $\$[$ we describe the effects that low and high altitude clouds would have on our calculations and in $\$ 7$ we discuss the implications of our results.

\section{Model}

Our calculations are based on the infrared orbital light curve model of GW04. These authors employ a linearized, analytic EBM to calculate the infrared flux emitted by a planet. This model assumes a single, uniform planetary albedo and parameterizes the thermal inertia and meridional heat transport across a planet's surface. The thermal effect of clouds is accounted for by subtracting a correction term from the outgoing flux (Caldeira \& Kasting, 1992). The time-dependent surface temperature distribution is described by a combination of Legendre polynomials and a Fourier series that are solutions to a diffusion equation with periodic temporal boundary conditions in a spherical coordinate system. The disk- and diurnally-averaged infrared flux for a 
prescribed viewing geometry is calculated along an entire orbit. As long as Eqn. 1 is satisfied, then diurnally averaging the infrared flux justifies the use of a single, average planetary albedo.

We consider only one set of Earth-like planetary parameters. Although such properties will undoubtedly vary amongst extra-solar planets, those planets with thermal properties similar to the Earth will be the most compelling targets of investigation. The thermal inertia of the surface $\left(8.34 \times 10^{7} \mathrm{~J} \mathrm{~m}^{-2} \mathrm{~K}^{-1}\right)$ and heat diffusion coefficient $\left(0.38 \mathrm{~W} \mathrm{~m}^{-2} \mathrm{~K}^{-1}\right)$ were chosen so that with an albedo $(A=0.3055)$, orbital semi-major axis $(\mathrm{a}=1 \mathrm{AU})$, and eccentricity $(\mathrm{e}=0.0167)$ of Earth, the model reproduces the meridional surface temperature distribution of the Earth as well as the seasonal temperature variation at several latitudes (GW04).

The orbital properties $(a, e$ and $i$ ) of a real planet can be determined by imaging or astrometry, but the thermal properties of the planet may not be uniquely determined by independent means. We discuss this scenario in $\$ 7$. Under the conditions of known orbital and thermal properties, the light curve is a function of the planet's obliquity $\left(\delta_{0}\right)$, the orbital longitude of inferior conjunction relative to the spring equinox $\left(L_{0}\right)$, and the orbital longitude of the apastron $\left(L_{a p}\right)$. If the orbit of the planet is nearly circular then the longitude of apoastron $L_{a p}$ (fixed here to $180^{\circ}$ ) is unimportant.

Ocean and atmospheric circulation and the thickness of an ocean's mixed layer may differ for Earth-like planets with obliquities that are significantly larger than $23.5^{\circ}$. Thus, the actual light curves of such planets would differ from those calculated with Earth-like thermal properties. We examined this effect by comparing general circulation model (GCM) runs for $\delta_{0}=85^{\circ}$ to 
our EBM calculations (Figure 1). The GCM used was the three-dimensional GENESIS 2 model (Williams \& Pollard, 2003) and the calculations were performed with $L_{0}=120^{\circ}$. The amplitude and general shape of the GCM light curves are nearly identical to those of the EBM. However, we find that the phase of the GCM light curves tend to lead those of the EBM by $\sim 35^{\circ}$, with the greatest differences occurring when the geometry of the system is such that the poles of the planet are pointed towards the observer (e. g. high obliquity and high inclination). This is likely due to the inclusion of polar sea ice in the GCM.

The exact origin of the offset in phase is uncertain, but we suspect that it involves effects of seasonal changes in cloud cover, sea ice or ocean circulation not included in the EBM. In $\$ 4$ we show that artificially adjusting the phase of the EBM light curve (for $i=60^{\circ}$ ) to better match the GCM calculations only slightly increases the probability of satellite detection. In \$5, we find that this phase lag does not affect the conclusion that an Earth-like planet with a lunarlike satellite produces light curves resembling those of an isolated planet with high obliquity (i.e. one with large amplitude, see GW04 for for a comparison of light curves from Earth-like planets with high and low obliquities). We are interested only in estimating the detectability of satellites and their gross effect on the interpretation of infrared light curves, rather than on detailed inferences about the climates of planets themselves and thus use the EBM to efficiently calculate light curves over a range of obliquity values.

Our formalism for calculating the outgoing infrared flux from a lunar-like satellite is also based upon the analysis in GW04. In the absence of an atmosphere or oceans, the energy-balance equation governing the temperature $(T)$ 
at a given point on the surface of a satellite with no latitudinal heat transfer is

$$
c \frac{\partial T}{\partial t}=S \cdot(1-A)-I(T)
$$

where time is denoted by $t$, incident stellar flux by $S$, albedo by $A$, and outgoing infrared flux $I(T) . S$ and $I(T)$ are calculated as functions of longitude and latitude on the surface of the satellite taking into account projection effects. To analytically solve this equation three assumptions are made. First $c$ is assumed constant in time and across the satellite surface. Second, the temperature dependence of the outgoing infrared flux is approximated as a linearized blackbody:

$$
I(T)=I(\bar{T}) \cdot(1+4(T-\bar{T}) / \bar{T})
$$

This follows the approach of classical energy balance models (North et al., 1981). Finally, we assume tidally-locked, synchronous rotation as is expected for large, collisionally-formed satellites (Gladman et al., 1996; Canup \& Agnor, 1998). These assumptions allow Fourier series solutions to Equation 2:

$$
T(\theta, \ell)=T_{0}(\theta)+\sum_{n=1}^{N}\left[a_{n}(\theta) \cos (n \ell)+b_{n}(\theta) \sin (n \ell)\right]
$$

where $T_{0}$ is the mean temperature for a given latitude $\theta$ and $\ell$ is the longitude on the surface of the satellite. $N$ is set to 10 as numerical tests show that larger values do not significantly change the final light curve. Substitution of Equations 3 and 4 into Equation 2 yields expressions for the Fourier 
coefficients:

$$
\begin{aligned}
& a_{n}(\theta)=\frac{a_{n}^{\prime}(B+\alpha)-b_{n}^{\prime} \alpha}{\alpha^{2}+(B+\alpha)^{2}} \\
& b_{n}(\theta)=\frac{a_{n}^{\prime} \alpha-b_{n}^{\prime}(B+\alpha)}{\alpha^{2}+(B+\alpha)^{2}}
\end{aligned}
$$

where $a_{n}^{\prime}, b_{n}^{\prime}, B$ and $\alpha$ are

$$
\begin{aligned}
& a_{n}^{\prime}=\int_{0}^{2 \pi} \frac{S(\ell, \theta)}{\pi} \cos (n \ell) d \ell \\
& b_{n}^{\prime}=\int_{0}^{2 \pi} \frac{S(\ell, \theta)}{\pi} \sin (n \ell) d \ell
\end{aligned}
$$

$$
\begin{gathered}
B=\frac{4 I(\bar{T})}{\bar{T}} \\
\alpha=\frac{\omega c}{2} \sqrt{n} .
\end{gathered}
$$

The infrared emission from the surface is calculated using Equation 3 and the total signal is determined by geometric projection of the hemisphere facing the observer.

For simplicity we assume that the orbit of the satellite is coplanar with that of the planet's orbit around the star and, because of synchronous rotation, the satellite has zero obliquity. Thus any variation in outgoing flux from the satellite is due to its finite heat capacity. The disk-averaged flux of the satellite is then independent of the location on its orbit around the planet and depends only on the geometric angle described by the star, satellite, and 
distant observer (Figure 2). Variability in the satellite signal is due to its observed phase, which changes with the orbital period of the planet. In $\$ 4$ we consider satellites that differ in size (but not surface properties) from the Moon.

A satellite larger than the Moon will retain heat for a longer time and be more likely to have active volcanism. This could make the satellite darker, as in the case of the lunar Mare. However, with an average albedo of 0.07 the Moon is already quite dark. Fresh basalt from active volcanism on the surface of a larger satellite would have little effect on its light curve.

\section{Example Light Curves and Observations}

Figure 2 illustrates how an Earth-Moon analog would appear at five evenlyspaced points in the system's orbit. Figure 3 plots the infrared light curves produced by this system. The bottom panel displays the disk-averaged flux, while the top shows light curves normalized to their respective means. This normalization (which is employed for all subsequent analysis) removes the radius of the planet as a degree of freedom in the model. The calculations were performed assuming an Earth "twin" $\left(\delta=23.45^{\circ}\right)$ with a satellite of radius, orbital period and albedo equal to that of the Moon $\left(0.273 \mathrm{R}_{\oplus}, 29.5\right.$ days and 0.073 respectively). For these and all simulations the coplanar orbits of the planet and satellite are inclined by $60^{\circ}$ with respect to the plane of the sky (the median value of an isotropic distribution). The dotted line in both panels is the contribution from the planet alone. The peak-to-peak amplitude of the satellite's signal (dash-dots in the bottom panel) is $55 \mathrm{~W} \mathrm{~m}^{-2}$, whereas the

planet's flux alone varies by only $4 \mathrm{~W} \mathrm{~m}^{-2}$. Because the thermal inertia of 
the satellite is low, it displays a larger relative infrared flux variation than that of the planet (Equation 10. However, because the satellite is smaller, the majority of the average flux originates from the planet.

The assignment of erroneous properties to a planet with an undetected satellite is illustrated in the top panel of Figure 3, where the dashed line is the best-fit planet-only model to the planet+satellite observations. This model is of a planet with Earth-like thermal properties and $\delta_{0}=75^{\circ}$ and $L_{0}=90^{\circ}$, i.e. quite different than the input values. The phase and amplitude of the light curve produced by these models are not independent and each depends on the obliquity and orbital longitude of inferior conjunction. Adjusting the orbital longitude of inferior conjunction could produce better agreement with phase, but would unacceptably decrease the amplitude of the light curve. In general large light curve amplitudes like those produced by Earth-like planets with Moon-like satellites can only be mimicked by single planets with very high obliquities and orbital geometries where the northern or southern hemisphere is pointed towards the observer.

An example of an observation scheme of five evenly-spaced measurements is shown in the top panel of Figure 3. In practice a minimum of three observations are required to confirm planetary status and reject background sources (Beichman et al., 2006). The TPF mission would conduct a minimum of 3-5 observations on each star during the first two years of a five-year mission. The remaining time would be spent on spectroscopic follow-up of a few dozen planets (Beichman et al., 2006). Spectroscopic observations could, in principle, be split into multiple integrations, however these would preferentially occur when the planet was near maximum elongation from the star, thus maximizing the 
S/N. Both a nulling interferometer (TPF-I, Darwin) or a coronographic imager (TPF-C) will obscure planets along some parts of orbits in the habitable zone (Brown, 2004). We thus consider two observing scenarios, one consisting of five observations at equal longitudinal intervals around the orbit, and a second consisting of 14 observation points restricted to half of the orbit furthest from the star. For example, $14 \times 2$-day integrations of 36 high-priority targets might be obtained in three years. Targets in the habitable zone of solar-mass stars (0.9-1.3 AU, Kasting et al. (1993)) will have orbital periods of 300-550 days and thus the accessible part of an orbit can be completely observed. For the 5-point "confirmation" observation scheme we assume a $\mathrm{S} / \mathrm{N}$ ratio of 10 per observation, the median value of the $\mathrm{S} / \mathrm{N}$ amongst all 234 stars for which $\mathrm{S} / \mathrm{N}>5$ is achievable for an Earth-sized planet in a 24-hour integration time. For the 14-point "characterization" scheme we assume a $\mathrm{S} / \mathrm{N}$ of 20, which will be the case for Earth-sized planets around the nearest $20 \%$ of the target stars (Beichman et al., 2006).

\section{Satellite Detection Limits}

We determine the minimum size of a Moon-like satellite that can be detected around an Earth-like planet whose gross thermal properties are outlined in $₫ 2$. We presume that the inclination of the planet's orbit with respect to the plane of the sky is independently measured by astrometry and use a single value of $60^{\circ}$. For these calculations the orbital and thermal parameters of the planet and the orbital period, albedo and thermal inertia of the satellite (assumed to be equal to that of the Moon) are held fixed, while $\delta_{0}, L_{0}$ and the satellite radius $\left(R_{s}\right)$ are allowed to vary. With the exception of cases with extremely 
high planetary obliquity where most of the planetary signal is at twice the orbital period (GW04), the signal from the planet and satellite will have the same period. There are then three unknowns $\left(\delta_{0}, L_{0}\right.$ and $\left.R_{s}\right)$ but only two measurable quantities; the amplitude and phase of the orbital signal. Thus it is not possible to uniquely disentangle the planetary and satellite signals. Instead, we define a satellite "detection" as the case where the observations cannot be accounted for by a planet-only model.

Our detection analysis is as follows: We generate an array of planetary light curves over the full ranges of $\delta_{0}\left[0-90^{\circ}\right]$ and $L_{0}\left[0-360^{\circ}\right]$. The satellite light curve for a given $R_{s}$ is calculated and added to each planet light curve in the array. Each total light curve is sampled at $N$ specified points according to either of the observing schemes described in 93 . Random noise with a given RMS is added to these measurements. We then perform an exhaustive search of planet-only light curves to find the minimum $\chi^{2}$-fit to the measurements. The analysis is repeated for different values of $R_{s}$. We interpret the confidence level $C$ associated with the value of $\chi^{2}$ and the number of degrees of freedom as the probability that the deviation from the planet-only model is due to the presence of a satellite. This is because $1-C$ is the probability that measurements of the light curve of the planet alone would result in a fit with a $\chi^{2}$ larger than the observed value, i.e. a false positive. We set the effective number of degrees of freedom to the minimum $\chi^{2}$ value associated with a best fit to $\mathrm{N}$ observations of the planet-only light curve. Although this minimum $\chi^{2}$ value is sensitive to the random noise characteristics of a given sampling, we find that a more robust calculation of the true $\chi^{2}$ minimum (i.e. averaging over a large number of random noise patterns) produces results that are in close 
agreement with this single noise characteristic approximation. Our minimum $\chi^{2}$ approximation produces an increase of nearly an order of magnitude in computational efficiency over the robust method.

We average $C$ over $L_{0}$, which cannot be independently determined for any system, but will have a uniform probability distribution. In addition, $C$ is averaged over all possible phases of the measurement scenarios, i.e., the longitudes at which the planet is observed are shifted by 2 degree increments over the orbital phase range $2 \pi / N$. This produces an average probability of detection $\langle C\rangle$ for a given $R_{s}$ and $\delta_{0}$. The planet's obliquity cannot be independently determined from the light curve of the planet+satellite, nor can it be assumed to have an isotropic distribution (Atobe \& Ida, 2007).

In Figures 4 and 5 we plot $\langle C\rangle$ vs. obliquity for several values of satellite radius (in units of planetary radius). For an Earth-sized planet, this range of radii corresponds to Vesta- to Mars-sized bodies. The two figures correspond to the "confirmation" (5 evenly-spaced observations at $\mathrm{S} / \mathrm{N}=10$ ) and "characterization" (14 observations at $\mathrm{S} / \mathrm{N}=20$ distributed around $50 \%$ of the planet's orbit) observation scenarios described in \$3. As the radius of the satellite increases the observations become increasingly inconsistent with a planet-only light curve, thus the probability of satellite detection increases. For satellites smaller than 0.33 planetary radii the probability of detection is lower in the "characterization" observing scheme. This is an effect of the observations being distributed around only $50 \%$ of the orbit. The incomplete phase sampling of this scheme does not capture the peak of the satellite flux which occurs at the longitude of superior conjunction. However for large satellites $\left(R_{s} \geq 0.33\right.$ planetary radii) the amplitude of the net light curve becomes 
so great that the satellite is detected even without complete phase coverage. The scatter in these probability curves is due to the stochastic noise added to each of the sample measurements. For both observation schemes $\langle C>$ is very weakly dependent on obliquity. These results show that a Moon-like satellite (0.27 Earth radii) would only be detectable with $\sim 30 \%$ confidence by either observation scheme. For the "confirmation" and "characterization" observing schemes a satellite would have to be 0.5 and 0.38 planetary radii respectively to be detected with $90 \%$ confidence. This corresponds to approximately Mars-sized satellites in orbit around an Earth.

In Figure 6] we consider how these results would change if a full threedimensional climate model were used to generate the planetary light curves. As previously stated, our EBM produces light curves that lag by $\sim 35^{\circ}$ relative to those of the GENESIS 2 GCM for obliquities of $23.5^{\circ}$ and $85^{\circ}$. To mimic the results of the GCM we offset the phase of the EBM light curves by $35^{\circ}$ and repeat the analysis of Figure 5. It would be computationally prohibitive to generate GCM light curves for the full range of obliquities that are included in this analysis, thus we approximate the GCM by applying this offset. We find that the probability of detection actually increases slightly with the phase-adjusted pseudo-GCM light curves (Figure 6). Thus, we conclude that our EBM results are conservative estimates for the probability of satellite detection.

\section{Errors Introduced By an Undetected Satellites}

As we showed in Figure 3, the application of planet-only models to a set of observations can result in mischaracterization of the Earth-like planet if a 
large satellite is present. Even if the gross thermal properties of the planet have been independently established, this will still produce erroneous values of $\delta_{0}$ and $L_{0}$. We describe this effect by recording the "true" $\left(\delta_{0}, L_{0}\right)$ pair for a given planet+satellite light curve and the $\left(\delta_{0}, L_{0}\right)$ pair of the best fit planet-only light curve as determined by a $\chi^{2}$ analysis. In some instances, a planet+satellite light curve can be fit with equally low $\chi^{2}$ by more than one planet-only light curve. For these cases, we choose the $\left(\delta_{0}, L_{0}\right)$ pairs that are closest to the true value. Figure 7 plots the direction and proportional magnitude of the error introduced by satellite confusion (The length of the vectors have been reduced for clarity). These simulations were run for an Earth-like planet with a satellite of radius equal to that of the Moon and (for clarity) no intrinsic noise added to the sample points. In nearly all cases, the presence of a lunar-like satellite makes an Earth-analog planet appear to have high $\left(>80^{\circ}\right)$ obliquity. Shifting the planetary light curve phases by $35^{\circ}$ to make them resemble the GCM results does not alter the tendency of the best-fit solutions towards high $\delta_{0}$.

The vectors in Figure 7 converge towards two values of $L_{0}\left(90^{\circ}\right.$ and $\left.270^{\circ}\right)$ because the planet-only light curve is in phase with that of the satellite at these $L_{0}$. For high obliquity planets, $L_{0}$ values of $90^{\circ}$ or $270^{\circ}$ translate to a planetary configuration in which the southern or northern hemispheres (respectively) face the observer. At high $\delta_{0}$, these are the only geometries that produce planet-only light curves with large amplitudes that can reasonably fit a planet+satellite system. Adjusting for the phase difference between the 
EBM and the GCM does effect these general trends in $L_{0}$. Instead of converging to $L_{0}$ values of $90^{\circ}$ and $270^{\circ}$, the GCM vector field reaches convergence at $L_{0}$ values that are shifted by $35^{\circ}$.

\section{The Effect of Clouds}

Clouds play an important role in the energy balance and climate of the Earth by reflecting sunlight and scattering and trapping long-wavelength radiation; they would presumably do so for Earth-like extra-solar planets as well. Although clouds represent a mean $20 \mathrm{~W} \mathrm{~m}^{-2}$ (8\%) gain in radiation for the Earth (Hartmann, 1994) we are concerned here only with their seasonal affect on the energy budget and disk-integrated outgoing radiation. These seasonal effects will be most prominent when one hemisphere is presented to the observer. On the Earth, low clouds (stratocumulus) produce a decrease in net radiation, while high clouds (cirrus) produce an increase (Hartmann, 1994).

We estimate the thermal effect of seasonal variability in stratocumulus clouds on the light curve of an Earth twin. We assume that these low-altitude clouds radiate at the same temperature as a clear atmosphere and surface, i.e. they produce no additional greenhouse effect. We use a cloud albedo of 0.7 (Hartmann, 1994). Comiso \& Stock (2001) estimated variation in cloud cover over the open ocean around Antarctica to vary by only $\pm 1 \%$ around a mean of $91 \%$. Seasonal variation of $\pm 10 \%$ around a mean of $80 \%$ was observed over the North Atlantic (Massons et al., 1998). We adopt a 20\% seasonal variation in the mean as a reasonable bracketing value. To produce the global mean Earth albedo of 0.31 , a dark ocean $(A=0.07)$ must be covered with $37 \%$ clouds. Although the average cloud cover on Earth is $\sim 60 \%$, only half of 
those are low-altitude stratocumulus (Minnis et al., 2002), a value in reasonable agreement with the calculated fraction of $37 \%$. A $\pm 20 \%$ fluctuation in the mean coverage produces an albedo variation of 0.047 . We assume that the albedo variation is uniformly distributed over each (northern/southern) hemisphere and that it varies sinusoidally in phase with the summer solstice. (This obviously produces a non-physical discontinuity at the equator which is unimportant for the purposes of estimating the magnitude of the effect of clouds). We examined the light curves of two cases: one in which an Earth twin is observed at a moderate inclination $\left(i=60^{\circ}\right)$ and the other in which a high-obliquity Earth is observed on an edge-on orbit $\left(i=90^{\circ}\right)$ so that one hemisphere is seen nearly pole-on (results not shown). In both cases the annual mean of the disk-averaged flux is slightly lower in the presence of low altitude clouds, but the amplitude and phase of the variation is essentially unchanged. Of course, pathological deviations from terrestrial patterns of cloudiness are possible on planets not quite like the Earth, but we have already shown with a GCM model (that includes parameterized cloudiness) that in at least the high obliquity regime, our conclusions are not significantly impacted.

For an Earth-like planet, high altitude clouds will cause a greenhouse effect whose net effect is to offset any decrease in infrared emission caused by their high albedo. Variation in the fraction of high-altitude cloud cover on time scales of less than $\sim 1$ day will be averaged over during typical integrations. We argue that variation in high cloud cover over longer time scales will be insignificant compared to estimated noise characteristics for a typical observation. High altitude clouds with a temperature of $210 \mathrm{~K}$ would emit $\sim 40 \%$ less radiation than the surface. With typical high cloud cover fractions of $\sim 30 \%$ 
(Minnis et al., 2002) and again assuming $\pm 20 \%$ fluctuations in coverage, we calculate that fluctuations in outgoing infrared flux due to high cloud variability will be on the order of a few percent (40\% of surface flux $\times 30 \%$ coverage $\times 20 \%$ variability), which would be unresolved by observations with an optimistic $\mathrm{S} / \mathrm{N}$ of 20. In a more extreme case (e.g. larger variability, greater mean surface coverage or lower emitting temperatures) the variability in a light curve due to high altitude clouds will act to further confuse its interpretation.

\section{Discussion}

The simulations presented here show that time-series infrared photometry by a TPF- or Darwin-like observatory would reveal only the very largest lunarlike satellites (Mars-sized) around Earth-analog planets, and then only if these Earth-like properties, i.e., the presence of oceans and/or a substantial atmosphere, have been established by independent means, e.g. spectroscopy or optical photometry. This conclusion holds for a wide range of planetary obliquity (Figs. 4,5],6), assuming that the approximations of the EBM do not grossly misrepresent the infrared light curve of a high-obliquity Earth-like planet (Fig. 11).

When interpreting infrared light curves, the presence of an undetected lunarlike satellite can suggest erroneous values of planetary obliquity and longitude of inferior conjunction. In the case of a planet with high thermal inertia, inferred values of $\delta_{0}$ near $90^{\circ}$ and $L_{0}$ values within $35^{\circ}$ of $90^{\circ}$ or $270^{\circ}$ may indicate the presence of a lunar-like satellite. This result is based on the assumption that the satellite and planetary orbits are coplanar, which may be not be the case for high obliquity planets. Kinoshita (1993) showed that the 
orbit of a satellite will stay in the equatorial plane of its host planet if the secular rate of change of the planet's obliquity is slower than the precessional speed of the satellite orbital plane. Thus satellites around planets that experienced rapid changes in obliquity [possibly by collisions as in the case of Uranus (Parisi \& Brunini, 1997)] would stay in their coplanar orbits.

If a satellite's orbit is non-coplanar then its rotation axis will be tilted with respect to the plane of the planet's orbit. This effectively causes a non-zero obliquity for the satellite which will modify the amplitude of the satellite's light curve but will not change its period. If this non-coplanar orbit precesses then the signal will change over a time-scale of many orbital periods.

If the thermal properties of a planet are not independently established via spectroscopy (Des Marais et al., 2002), visible-wavelength detection of glint from an ocean or significant polarization of visible reflectance (Williams \& Gaidos, 2008; McCullough, 2008), then the flux from an unresolved lunar-like satellite can induce serious errors. If the measurements are modeled with the several free parameters (e.g. $\delta_{0}, L_{0}, A, c$ and efficiency of meridional heat transport) then a set of planet+satellite measurements can be satisfactorily fit by a planet-only light curve. For instance, the peak-to-peak light curve amplitude from a system with a large, unresolved lunar-like satellite around an Earthanalog planet can be fit by a planet with low thermal inertia and drastically different $\delta_{0}$ and $L_{0}$, implying a planet more akin to Mars than Earth. Such an erroneous inference would impact the determination of the frequency of habitable planets. This reinforces the need for multiple wavelength observations including spectroscopy and photometry to disambiguate the characterization of extra-solar terrestrial planets (Traub et al., 2006; Beichman et al., 2006). 
Where, optimistically, the thermal properties of a planet are known and its satellite is Mars-sized, the existence of a satellite may be inferred from infrared data. Such a discovery would provide information about the collisional and kinematic evolution of the parent planet. In addition, a large satellite could be a potential indicator of habitability, as the presence of the Moon is

known to stabilize the obliquity and climate of the Earth (Laskar et al., 1993).

\section{Acknowledgments}

This work has been supported by NASA Terrestrial Planet Finder Foundation science award NNG04GL48G. We thank the anonymous reviewers for their helpful comments. 


\section{REFERENCES}

Atobe, K. and Ida, S. (2007) Obliquity evolution of extrasolar terrestrial planets. Icarus, 188, 1-17.

Beichman, C., Lawson, P., Lay, O., Ahmed, A., Unwin, S., and Johnston, K. (2006) Status of the terrestrial planet finder interferometer (TPF-I). SPIE Proceedings, 6268, 62680-S1.

Brown, R. A. (2004) Obscurational completeness. ApJ, 607, 1003-1013.

Caldeira, K. and Kasting J. F. (1992) Susceptibility of the early Earth to irreversible glaciation caused by carbon dioxide clouds. Nature, 359, 226228.

Canup, R. M. and Agnor, C. (1998) Accretion of terrestrial planets and the Earth-Moon system. In Origin of the Earth and Moon, edited by K. Righter and R. Canup, LPI, Houston, pp. 113-129.

Comiso, J. C. and Stock, L. V. (2001) Studies of Antarctic cloud cover variability from 1981 to 1999. Proceedings of IGARSS-2004, 4, 1782-1785.

Cowan, N. B., Agol, E. and Charbonneau, D. (2007) Hot nights on extrasolar planets: mid-infrared phase variations of hot Jupiters. MNRAS, 379, 641646.

Des Marais, D. J. and 9 co-authors. (2002) Remote sensing of planetary properties and biosignatures on extrasolar terrestrial planets. Astrobiology, 2, 153-181.

Ford, E. B., Seager, S., and Turner, E. L. (2001) Characterization of extrasolar terrestrial planets from diurnal photometric variability. Nature, 412, 885887. 
Fridlund, C. V. M. (2000) Darwin - The Infrared Space Interferometry Mission. ESA Bulletin, 103, 20-25.

Gaidos, E. and Williams, D. M. (2004) Seasonality on terrestrial extrasolar planets: inferring obliquity and surface conditions from infrared light curves. New Astronomy, 10, 67-78.

Gillon, M., Courbin, F., Magain, P., and Borguet, B. (2005) On the potential of extrasolar planet transit surveys. $A \mathscr{E} A, 442,731-744$.

Gladman, B., Dane Quinn, D., Nicholson, P., and Rand, R. (1996) Synchronous locking of tidally evolving satellites. Icarus, 122, 166-192.

Harrington, J. and 7 co-authors. (2006) The phase-dependent infrared brightness of the extrasolar planet $\nu$ Andromedae b. Science, 314, 623-626.

Hartmann, W. K. (1986) Moon origin - The impact trigger hypothesis. In Origin of the Moon, edited by W. K. Hartmann, R. J. Phillips, and G. J. Taylor, LPI, Houston, pp. 579-608.

Hartmann, D. L. (1994) Global Physical Climatology. Academic Press, San Diego.

Ida, S., Canup, R. M., and Stewart, G. R. (1997) Lunar accretion from an impact-generated disk. Nature, 389, 353-357.

Kasting, J. F., Whitmire, D. P., and Reynolds, R. T. (1993) Habitable zones around main sequence stars. Icarus, 101, 108-128.

Kinoshita, H. (1993) Motion of the orbital plane of a satellite due to a secular change of the obliquity of its mother planet. CeMDA, 57, 359-368.

Knutson, H. A., Charbonneau, D., Allen, L. E., Fortney, J. J., Agol, E., Cowan, N. B., Showman, A. P., Cooper, C. S., and Megeath, S. T. (2007) A map of the day-night contrast of the extrasolar planet HD 189733b. Nature, 447, 
183-186.

Lane, A. P. and Irvine, W. M. (1973) Monchromatic phase curves and albedos for the lunar disk. AJ, 78, 267-277.

Laskar, J., Joutel, F., and Robutel, P. (1993) Stabilization of the Earth's obliquity by the Moon. Nature, 361, 615-617.

Lissauer, J. J., Dones, L., and Ohtsuki, K. (2000) Origin and evolution of terrestrial planet rotation, In Origin of the Earth and Moon, edited by K. Righter and R. Canup, LPI, Houston, pp. 101-112.

Massons, J., Domingo, D., and Lorente, J. (1998) Annales Geophysicae, 16, $331-341$.

McCullough, P. R. (2008) Models of Polarized Light from Oceans and Atmospheres of Earth-like Extrasolar Planets. submitted to ApJ, arXiv:astro-ph/0610518.

Minnis, P., Young, D. F., Wielicki, B. A., Sun-Mack, S., Trepte, Q. Z., Chen, Y., Heck, P., and Dong, X. (2002) A Global Cloud Database from VIRS and MODIS fro CERES. SPIE 3rd International Asia-Pacific Environmental Remote Sensing Symposium, Remote Sensing of the Atmosphere, Ocean, Environment, and Space. Hangzhou, China. October 23-27, 2002.

Muller, T. G., and Lagerros, J. S. V. (1998) Asteroids as far-infrared photometric standards for ISOPHOT. A\&A, 338, 340-352.

North, G. R., Cahalan, R. F., and Coakley, J. A. (1981) Energy balance climate models. Rev. Geophys. Space Phys., 19, 91-121.

Parisi, M. G. and Brunini, A. (1997) Constraints to Uranus' Great Collision-II PESS, 45, 181-187. 
Pritchard, M. E. and Stevenson, D. J. (2000) Thermal aspects of a lunar origin by giant impact, In Origin of the Earth and Moon, edited by K. Righter and R. Canup, LPI, Houston, pp. 179-196.

Rivera, E. J. and 8 co-authors. (2005) A $\sim 7.5 M_{\oplus}$ planet orbiting the nearby star GJ 876. ApJ, 634, 625-640.

Selsis, F. (2004) The Atmosphere of Terrestrial Exoplanets: Detection and Characterization. In Extrasolar Planets: Today and Tomorrow, ASP Conference Proceedings, edited by J. Beaulieu, A. Lecavelier des Etangs and C. Terquem, Institut D'Astrophysique de Paris, France, vol. 321, p. 170.

Traub, W. A. and 25 co-authors. (2006) TPF-C: status and recent progress. SPIE Proceedings, 6268, 62680T.

Udry, S. and 10 co-authors. (2007) The HARPS search for southern extrasolar planets XI. Super Earths $\left(5 \& 8 M_{\oplus}\right.$ ) in a 3 -planet system. $A \mathscr{E} A, 469$, L43-L47.

Williams, D. M., and Pollard, P. (2003) Extraordinary climates of Earth-like planets: three-dimensional climate simulations at extreme obliquity. Inter. J. Astrobio, 2, 1-19.

Williams, D. M. and Gaidos, E. J. (2008) Detecting the glint of starlight on the oceans of distant planets. Icarus, 195, 927-937. 


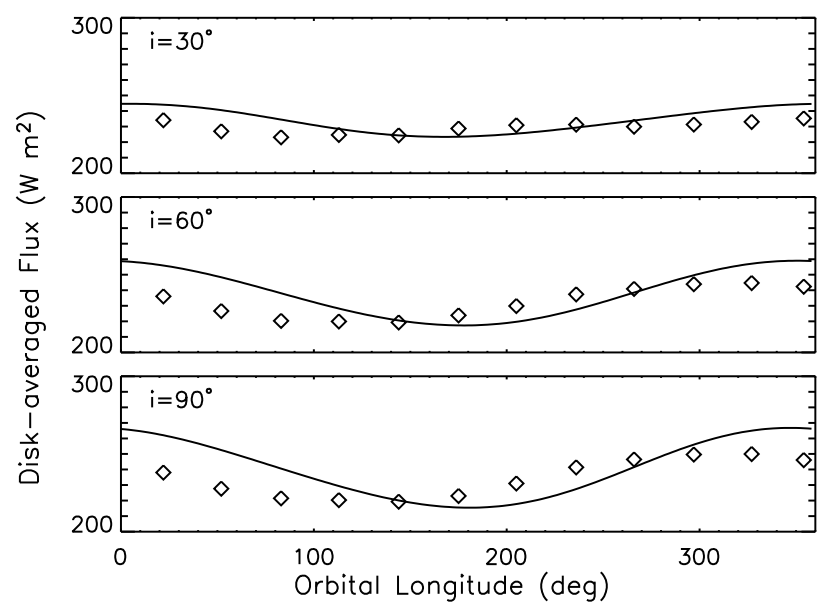

Figure 1. Calculated EBM (solid) and GCM (diamonds) light curves of a high-obliquity $\left(\delta_{0}=85^{\circ}\right)$ planet with Earth-like thermal properties. Light curves are shown for three values of inclination. $L_{0}=120^{\circ}$ (defined with respect to the vernal equinox) is used for both models. The two models are in close agreement regarding the amplitude of the planetary signal, however the phase of the EBM calculations tend to lag by $\sim 35^{\circ}$. 


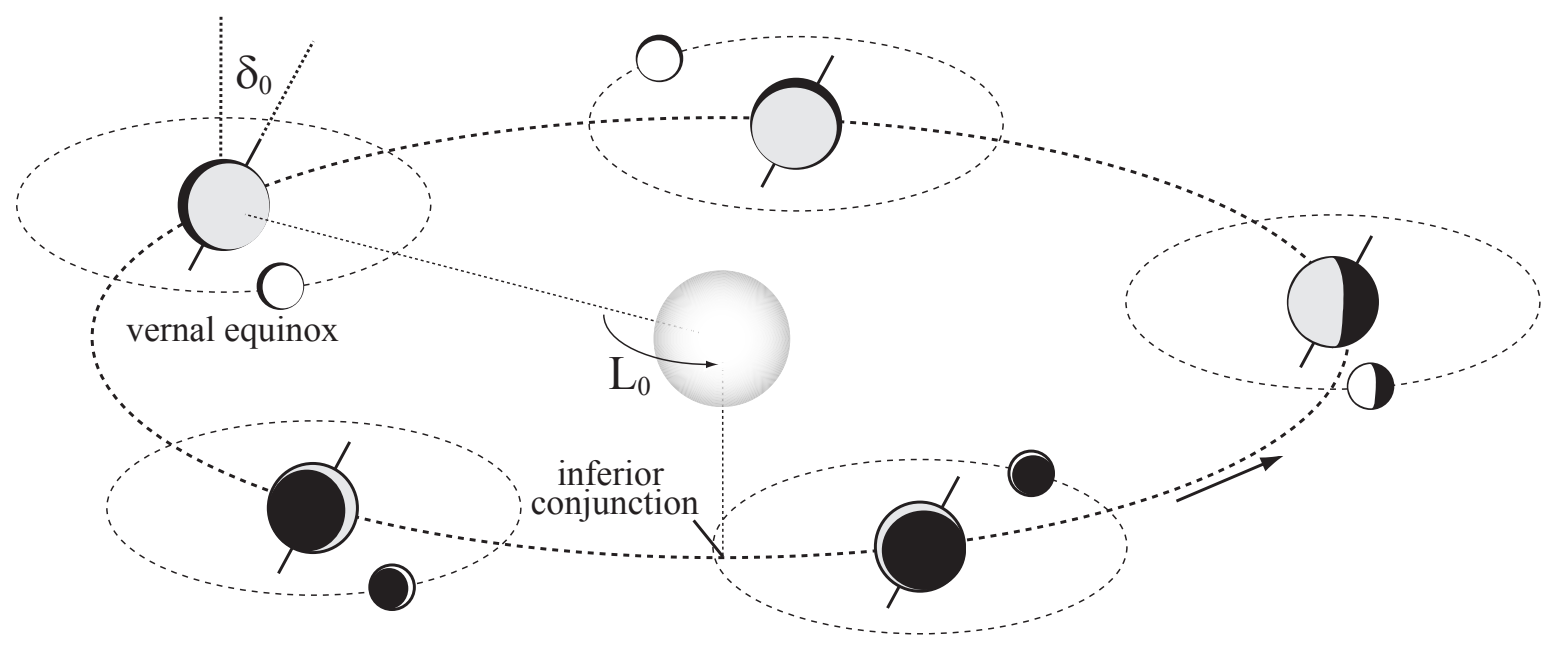

Figure 2. Planet and satellite at five uniformly-spaced orbital longitudes. The vernal equinox corresponds to $L=0$ and inferior conjunction to $L=L_{0}$. The disk-averaged flux from the Earth-like planet, which has a high thermal inertia, varies only with seasonal surface temperature differences between the two hemispheres. The disk-average flux from the Moon-like satellite, which has a low thermal inertia, depends on its observed phase. The observed phase of the satellite depends only on its orbit around the star, not around the planet. 

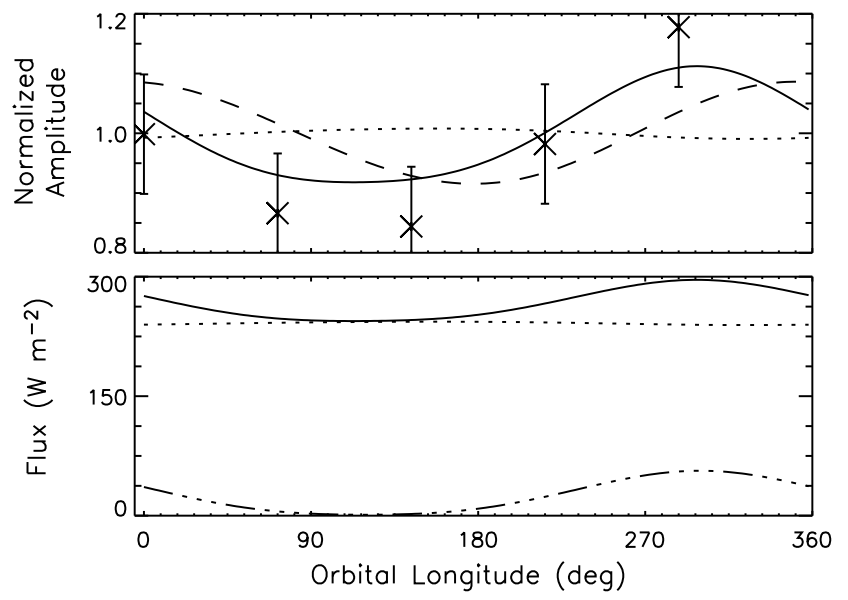

Figure 3. Simulated light curves and observations of an EarthMoon "twin". The bottom panel shows disk-averaged flux while the top displays the normalized light curves. In both panels the dotted curve is the planetary seasonal flux and the solid curve is the "true" planet+satellite light curve. The dash-dot curve in the bottom panel is the satellite contribution. In the top panel the sample points are indicated by X's with one-sigma error bars for a $\mathrm{S} / \mathrm{N}=10$. The best fit, planet-only light curve to the sample points is represented by the dashed line. The top panel light curves have been normalized by their mean to remove any dependence on the radius of the planet. 


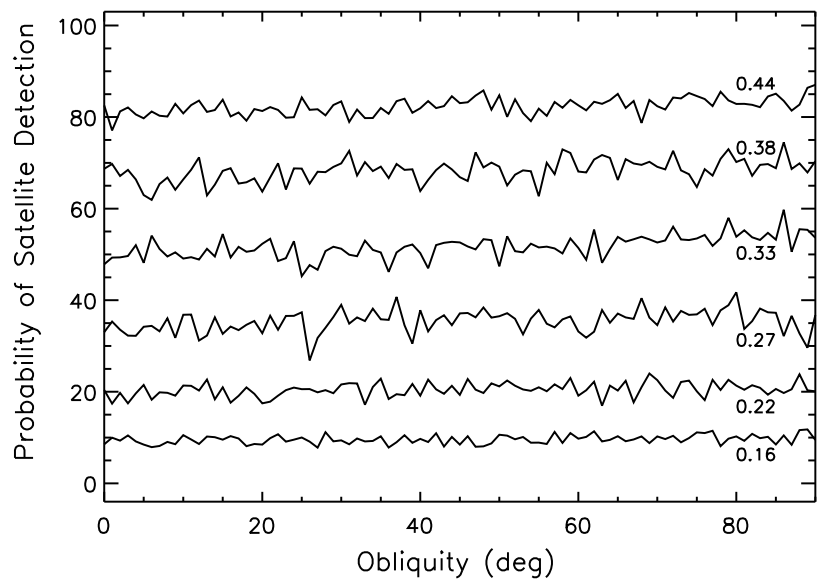

FigurE 4 . The $L_{0}$ averaged probability of satellite detection as a function of planetary obliquity for a range of satellite radii $(0.16,0.22,0.27,0.33,0.38$ and 0.44 planetary radii). The radius of the Moon is $0.27 R_{\oplus}$. These simulations were performed with 5 evenly-spaced observations and a $\mathrm{S} / \mathrm{N}$ of 10 . As the radius of the satellite increases it becomes increasingly difficult to explain the sample measurements with a planet-only light curve, thus the probability of detection increases. 


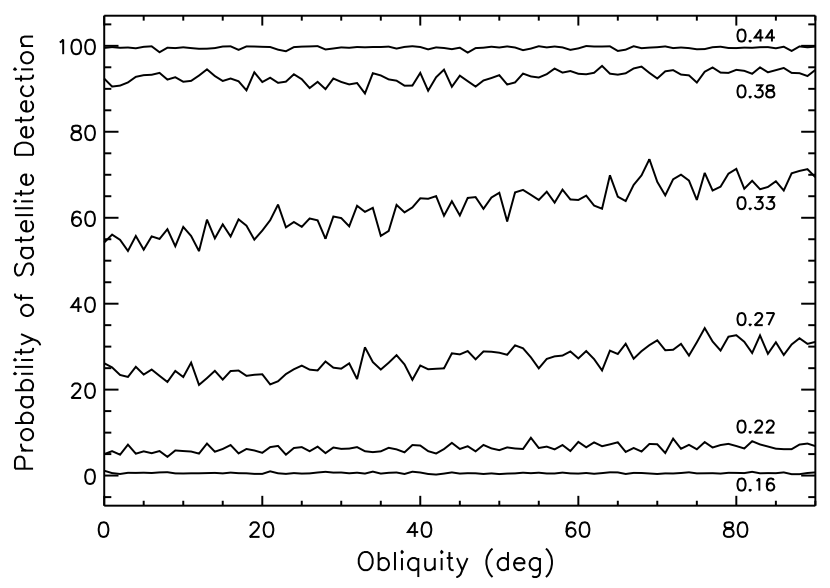

Figure 5. Similar to Figure 4, except for the "characterization" observing scheme: 14 sample points with a $\mathrm{S} / \mathrm{N}$ of 20 , distributed around $50 \%$ of the planet's orbit. Although the S/N is higher than the observations of Figure 4, the distribution of sample points around only $50 \%$ of the orbit makes it difficult to detect satellites of small radii ( $\leq 0.33$ planetary radii). 


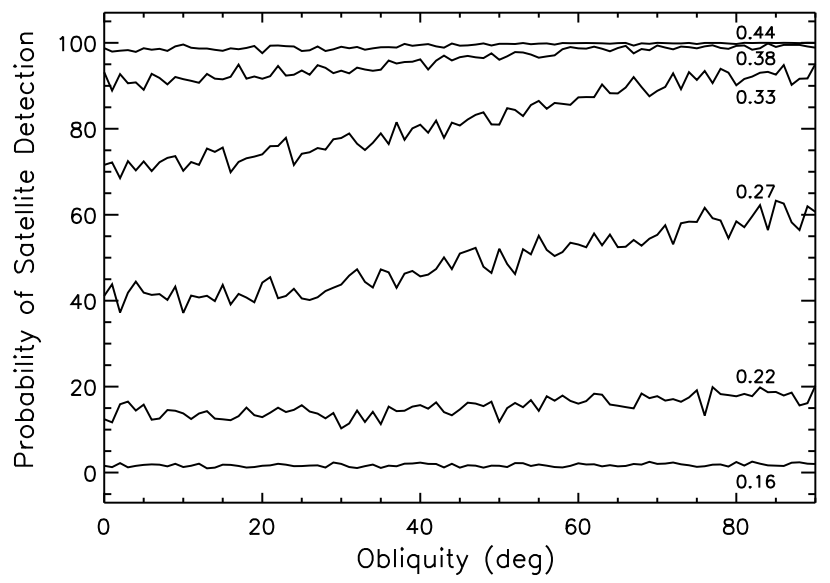

Figure 6. Similar to Figure [5, except that a $35^{\circ}$ phase shift has been applied to all EBM planetary light curves so that they agree with the GENESIS 2 GCM. Using these pseudo-GCM light curves the detection probability actually increases relative to the EBM case (Figure 51). This suggests that the EBM places a lower limit on the probability of satellite detection. 


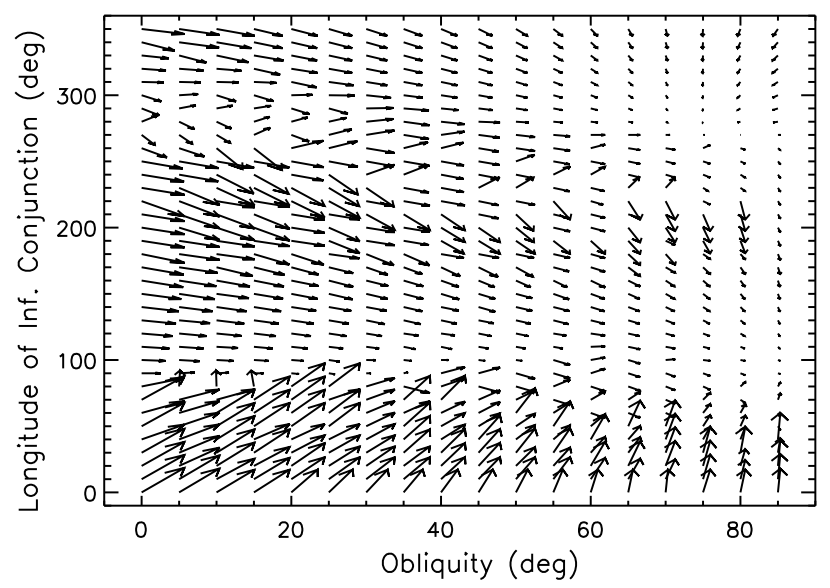

Figure 7 . Error induced in the light curve analysis by an undetected satellite. The vectors point from the true $\left(\delta_{0}, L_{0}\right)$ pair that is used to generate the planet+satellite light curve, towards the best fit planet-only $\left(\delta_{0}, L_{0}\right)$ pair. For clarity, the length of the vectors have been scaled to the amount of induced error. Their endpoints do not actually fall on the best-fit $\left(\delta_{0}, L_{0}\right)$ values. Nearly all best fit values cluster at high obliquity and $L_{0}$ values of $\sim 90^{\circ}$ or $\sim 270^{\circ}$. 